\title{
On the set of divisors of an integer
}

H. Maier ${ }^{1}$ and G. Tenenbaum ${ }^{2}$

Dedicated to Professor Pál Erdös

on the occasion of his 70-th birthday

1 Department of Mathematics, University of Michigan, Ann Arbor, MI 48109, USA

${ }^{2}$ UER Sciences Mathématiques, Université de Nancy I, Boîte Postale 239

F-54506 Vandoeuvre les Nancy Cedex, France

\section{Introduction}

Albeit the distribution of the prime factors of an integer determine in principle that of the divisors, it is often very hard in practice to describe the latter using currently available information about the former. A typical example is supplied by the 45 year old conjecture of Erdös [1] stating that almost all integers possess at least a pair of divisors $d, d^{\prime}$, with the property that

$$
d<d^{\prime} \leqq 2 d .
$$

This problem and several other questions connected with the distribution and the propinquity of divisors have been studied recently $[2,4,8,12-14]$. Another related topic is the theory of Hooley's $\Delta$-function which has gained importance in several applications to different branches of Number Theory [11]. This function is defined by

$$
\Delta(n):=\sup _{u} \operatorname{card}\{d: d \mid n, u<d \leqq \mathrm{e} u\}, \quad(n=1,2, \ldots) .
$$

The normal and average orders of $\Delta$ have been investigated by Hall and Tenenbaum in $[9,10]$. The second paper also deals with the generalized $\Delta_{r}$-functions (see [11]) which are of specific interest in some applications. The best known results are

$$
x \log \log x \ll \sum_{n \leqq x} \Delta(n) \ll x(\log x)^{\alpha}
$$

with $\alpha=0.21969$, and

$$
\Delta(n) \ll(\log n)^{\beta}, \quad \text { (p.p.) }
$$

for any $\beta>\log 2(1-1 / \log 3)=0.06221 \ldots$. Here and throughout the paper we use the notation p.p. (presque partout) to indicate that a relation holds for almost all integers - that is in a sequence of asymptotic density 1. 
In this paper, our aim is to prove a strong form of Erdös's conjecture and to give a lower bound for the normal order of Hooley's function. We now state our main result.

Theorem 1. Let $E(n)$ be the infimum of the numbers $\log \left(d^{\prime} / d\right)$, when $d\left|n, d^{\prime}\right| n$, $d<d^{\prime}$. If $\xi(n)$ is any function tending to infinity, we have

$$
E(n) \leqq(\log n)^{1-\log 3} \exp \{\xi(n) \sqrt{\log \log n}\}, \quad \text { (p.p.). }
$$

We first make some historical comments. Originally the first named author gave a proof of the slightly weaker result

$$
(\forall \varepsilon>0), \quad E(n) \leqq(\log n)^{1-\log 3+\varepsilon}, \quad \text { (p.p.). }
$$

His approach was indirect: he first established a comparison theorem which enables one to translate the problem into a purely probabilistic statement, namely that a certain integral is asymptotically $1+o(1)$. He then used an iterative procedure that yielded the desired estimate. Retaining the basic idea of this last step, the second author provided a number theoretical demonstration, along lines similar to those of $[7,14]$. We present this second proof here. Ignoring the slight improvement on the final result, it has the advantage of being easier in principle, and considerably shorter.

The result stated in Theorem 1 is nearly best possible. As shown by a theorem of Erdös and Hall [2] not only the exponent 1- $\log 3$ cannot be improved but, also, the function $\xi(n)$ cannot be taken tending to $-\infty$ as fast as $-c \sqrt{\log \log \log \log n}$.

An easy heuristic argument explains why the exponent $1-\log 3$ has to be expected. The number of distinct ratios $d^{\prime} / d$, when $d\left|n, d^{\prime}\right| n$, is equal to

For all $n$ we have

$$
U(n):=\prod_{p^{v} \| n}(2 v+1)
$$

$$
3^{\omega(n)} \leqq U(n) \leqq 3^{\Omega(n)},
$$

where $\omega(n)$ (resp. $\Omega(n)$ ) denotes the number of prime factors of $n$ counted without (resp. with) multiplicity. By the Hardy-Ramanujan Theorem, both functions $\omega(n)$ and $\Omega(n)$ have normal order $\log \log n$. Thus, assuming smooth distribution of the $\log \left(d^{\prime} / d\right)$ in $[-\log n, \log n]$, any sub-interval of length $\lambda$ should normally contain as its appropriate share

$$
\lambda U(n) /(2 \log n)=\lambda(\log n)^{\log 3-1+o(1)}
$$

distinct values $\log \left(d^{\prime} / d\right)$. We apply this to an interval centered at the origin.

Theorem 2. Let $\gamma<-\log 2 / \log (1-1 / \log 3)=0.28754 \ldots$. Then

$$
\Delta(n)>(\log \log n)^{\gamma}, \quad \text { (p.p.). }
$$




\section{Two lemmas}

We shall use in the sequel the following lemmas. They can both be proven elementarily. The first is a weakening of a theorem of Halberstam and Richert [6] generalizing a result of Hall. The second is established in [5] and, in a stronger version, in [14].

Lemma 1. Let $f$ be a non negative multiplicative function such that for all primes $p$

$$
0 \leqq f\left(p^{v}\right) \leqq \lambda_{1} \lambda_{2}^{v}, \quad(v=1,2, \ldots),
$$

where $0<\lambda_{1}, 0<\lambda_{2}<2$. Then for $x \geqq 1$

$$
\sum_{n \leqq x} f(n) \ll_{\lambda_{1}, \lambda_{2}} x \prod_{p \leqq x}\left(1-p^{-1}\right) \sum_{\nu=0}^{\infty} f\left(p^{v}\right) p^{-v} .
$$

Lemma 2. For $2 \leqq u \leqq v \leqq x$, we have

$$
\operatorname{card}\left\{n \leqq x: \prod_{p \leqq u, p^{v|| n}} p^{v} \geqq v\right\} \ll x \exp \left(-c \frac{\log v}{\log u}\right),
$$

where $c$ is a positive absolute constant.

\section{Proof of Theorem 1}

We first introduce some notation.

The letter $p$ always denotes a prime number. We set $\eta=\eta(x)$ $=(\log x)^{1-\log 3} \exp \{\xi(x) \sqrt{\log \log x}\}, \quad \varepsilon=\varepsilon(x)=\xi(x)(\log \log x)^{-\frac{1}{2}}, \quad \varepsilon_{1}=\varepsilon_{1}(x)=\varepsilon / 10$, $L=\left[\left(1-2 \varepsilon_{1}\right) \log \log x\right], \quad M=\left[\left(1-\varepsilon_{1}\right) \log \log x\right], \quad r_{k}=\exp \exp k,(L \leqq k \leqq M) . \mathrm{We}$ suppose that $\xi(x)$ tends to $+\infty$ so slowly that $\varepsilon(x) \rightarrow 0$.

For every integer $n \leqq x$, we define $n_{k}=\prod_{p \mid n, p<r_{k}} p$ and we denote by $\lambda(n)$ the Lebesgue measure of the set

$$
\bigcup_{d d^{\prime} \mid n}\left(\log \left(d^{\prime} / d\right)+[-\eta, \eta]\right)
$$

For real $\theta$ and square free $n$ we write

$$
S(\theta ; n)=\sum_{d d^{\prime} \mid n}\left(d^{\prime} / d\right)^{i \theta}=\prod_{p \mid n}(1+2 \cos (\theta \log p)) .
$$

Finally, the notation p.p.x means: for a set of cardinality $x+o(x)$ of integers $\leqq x$.

The basic idea of the proof is to show that $\lambda\left(n_{k}\right)$ is fairly large p.p.x. This leaves many possibilities for those prime factors of $n$ larger than $r_{k}$ to lie close to a ratio $d^{\prime} / d$ with $d d^{\prime} \mid n_{k}$. In other words this means that if $n_{k}$ does not satisfy the conjecture, then the conditional probability that, for small $l, n_{k+l}$ still does not satisfy it is not to close to 1 . The proof is then completed by inductive use of this upper bound.

We start with a lower estimate for $\lambda(n)$ derived from Fourier Analysis. 
Lemma 3. We have, for square free $n$,

$$
\lambda(n) \geqq 3^{2 \omega(n)}\left(2 \pi \int_{-1 / \eta}^{1 / n} S(\theta ; n)^{2} d \theta\right)^{-1} .
$$

Proof. Set $F(z)=\sum_{d d^{\prime} \mid n, \log \left(d^{\prime} / d\right) \leqq z} 1$. Then $\lambda(n)$ is the measure of the set of those $z$ for which $F(z+\eta)-F(z-\eta) \neq 0$. Plainly

$$
\begin{aligned}
F(z+\eta)-F(z-\eta) & \leqq 2 \int_{-\infty}^{+\infty}\left(\frac{\sin ((u-z) / 2 \eta)}{(u-z) / 2 \eta}\right)^{2} d F(u) \\
& =2 \eta \int_{-1 / \eta}^{1 / \eta} e^{i \theta z}(1-|\theta \eta|) S(\theta ; n) d \theta
\end{aligned}
$$

by Parseval's formula. A second application of this formula implies

$$
\int_{-\infty}^{+\infty}(F(z+\eta)-F(z-\eta))^{2} d z \leqq 8 \pi \eta^{2} \int_{-1 / \eta}^{1 / \eta}(1-|\theta \eta|)^{2} S(\theta ; n)^{2} d \theta .
$$

This gives the result stated by the Cauchy-Schwarz inequality:

$$
\left(2 \eta 3^{\omega(n)}\right)^{2}=\left(\int_{-\infty}^{+\infty}(F(z+\eta)-F(z-\eta)) d z\right)^{2} \leqq \lambda(n) \int_{-\infty}^{+\infty}(F(z+\eta)-F(z-\eta))^{2} d z .
$$

Lemma 4. Let $0<\alpha<1$ and let $w(x)$ be any function tending to infinity. For each $k, L \leqq k \leqq M$, we have uniformly in $s, w(x) \leqq s \leqq k$,

Moreover,

$$
\omega\left(n_{k} / n_{k-s)} \geqq \alpha s, \quad \text { (p.p. } x\right) \text {. }
$$

$$
\left.\omega\left(n_{k}\right) \geqq\left(1-\varepsilon_{1}\right) k, \quad \text { (p.p. } x\right) .
$$

Proof. The second inequality readily follows from the Turán-Kubilius inequality. To prove the first, we use Lemma 1. The number of exceptional integers up to $x$ does not exceed

$$
\sum_{w(x) \leqq s \leqq k} \sum_{n \leqq x} \alpha^{\omega\left(n_{k} / n_{k}-s\right)-\alpha s} \ll x \sum_{s \geqq w(x)} e^{-Q(\alpha) s}
$$

where $Q(\alpha)=\alpha \log \alpha-\alpha+1>0$. This is sufficient.

Lemma 5. Let $k$ and $w(x)$ be as in Lemma 4. We have

$$
\left.\int_{-1 / \eta}^{1 / \eta} S\left(\theta ; n_{k}\right)^{2} d \theta \leqq 3^{2 \omega\left(n_{k}\right)} e^{-k} w(x), \quad \text { (p.p. } x\right) \text {. }
$$

Corollary. Under the same hypothesis, we have

$$
\left.\lambda\left(n_{k}\right) \geqq e^{k} / w(x), \quad \text { (p.p. } x\right) .
$$

Proof of the Lemma. Since $\left|S\left(\theta ; n_{k}\right)\right| \leqq 3^{\omega\left(n_{k}\right)}$, the desired estimate is valid for all $n$ for the sub-range $|\theta| \leqq e^{-k} w(x)$. We may therefore suppose $|\theta|>e^{-k} w(x)$. Set $\omega_{\theta}(n)=\sum_{p|n, \log p \leqq 1 /| \theta \mid} 1$. 
By Lemma 1, we have for $y, z>0$

$$
\begin{aligned}
& \sum_{n \leqq x} S\left(\theta ; n_{k}\right)^{2} z^{\omega\left(n_{k}\right)} y^{\omega_{\theta}(n)} \\
& \quad \ll x \exp \left\{\sum_{p \leqq e^{1 /|\theta|}} \frac{9 y z}{p}+\sum_{e^{1 /|\theta|<p \leqq r_{k}}} \frac{z(1+2 \cos (\theta \log p))^{2}}{p}-k\right\} \\
& \quad \ll x \exp \left\{3 z(3 y-1) \log ^{+}(1 /|\theta|)+(3 z-1) k+O_{y, z}(\log \log (3+|\theta|))\right\},
\end{aligned}
$$

the second sum over $p$ being estimated, using the Prime Number Theorem, as explained in [3; Lemma 2]. Next, we select $y=z=1 / 3$, and use Lemma 4 in the form

We obtain

$$
\begin{aligned}
\left.\omega\left(n_{k}\right)-\omega_{\theta}(n) \geqq \alpha(k-\log (1 /|\theta|)), \quad\left(e^{-k} w(x)<|\theta| \leqq 1\right), \quad \text { (p.p. } x\right) \\
\left.\omega\left(n_{k}\right) \geqq\left(1-\varepsilon_{1}\right) k, \quad \text { (p.p. } x\right) .
\end{aligned}
$$

$$
\sum_{n \leqq x} S\left(\theta ; n_{k}\right)^{2} 3^{-2 \omega\left(n_{k}\right)} \ll\left\{\begin{array}{l}
x\left(e^{k}|\theta|\right)^{-\alpha \log 3}, \quad\left(e^{-k} w(x)<|\theta| \leqq 1\right), \\
x 3^{-\left(1-\varepsilon_{1}\right) k}(\log (3+|\theta|))^{A}, \quad(1<|\theta| \leqq 1 / \eta),
\end{array}\right.
$$

where the dash indicates that the sum is restricted to a set of integers $\leqq x$, independent of $\theta$, of cardinality $x+o(x)$, and where $A$ denotes an absolute constant.

We now fix $\alpha$ in the range $1 / \log 3<\alpha<1$ and perform the integration in $\theta$. This yields

$$
\sum_{n \leqq x}^{\prime} \int_{e^{-k} w(x)<|\theta| \leqq 1 / \eta} S\left(\theta ; n_{k}\right)^{2} 3^{-2 \omega\left(n_{k}\right)} d \theta \ll x e^{-k}
$$

Indeed, the contribution of the first range is easily seen to be of the desired order and that of the second is

$$
\ll x 3^{-\left(1-\varepsilon_{1}\right) k} \eta^{-1}(\log \log x)^{A}=o\left(x e^{-k}\right) .
$$

This completes the proof.

Proof of Theorem 1. Let $E_{k}$ be the number of integers $n \leqq x$ having the following property

$$
d d^{\prime}\left|n_{k}, \quad d \neq d^{\prime} \Rightarrow\right| \log \left(d^{\prime} / d\right) \mid>\eta(x)
$$

We denote by $E_{k}^{\prime}$ the number of those $n$ counted in $E_{k}$ which satisfy the three extra conditions

(a) $\log n_{k} \leqq e^{k} w(x)$,

(b) $\omega\left(n_{k}\right) \leqq 2 k$,

(c) $\lambda\left(n_{k}\right) \geqq e^{k} / w(x)$.

By Lemma 2, the Turán-Kubilius inequality and Corollary to Lemma 5 , we see that for each $k, L \leqq k \leqq M$,

$$
E_{k} \leqq E_{k}^{\prime}+o(x)
$$

We shall prove by contradiction that $E_{M}=o(x)$. To this end, we consider the number $F_{k}$ of those integers $n$ counted in $E_{k}^{\prime}$ which have two prime factors $p$ and $q$ such that 
(i) $2 e^{k} w(x)<\log p \leqq 3 e^{k} w(x)$,

(ii) $\log p<\log q \leqq \log p+e^{k} w(x)$,

(iii) $\log q-\log p \in \bigcup_{d d^{\prime} \backslash n_{k}}\left(\log \left(d^{\prime} / d\right)+[-\eta, \eta]\right)$.

The last condition ensures that $\left|\log \left(p d^{\prime} / q d\right)\right| \leqq \eta(x)$ for some $d, d^{\prime}, d d^{\prime} \mid n_{k}$, so that $n$ is certainly not counted in $E_{k+l}$, with $l=1+[\log (4 w(x))]$. Thus, $F_{k} \leqq E_{k}$ $-E_{k+l}$. Denote by $m_{k}$ an integer equal to $n_{k}$ for some $n$ counted in $E_{k}^{\prime}$, and by $b$ an integer all of whose prime factors are $>\exp \left(4 e^{k} w(x)\right)$. By the sieve

$$
F_{k} \geqq \sum_{\substack{t m_{k} p q b \leqq x \\ t \mid m_{k}}}^{*} 1 \gg \sum_{m_{k}^{2} p q \leqq x}^{*} \frac{x e^{-k}}{w(x) p q \varphi\left(m_{k}\right)}
$$

where the star indicates that $p$ and $q$ satisfy (i), (ii), (iii) above with $m_{k}$ in place of $n_{k}$. The condition $m_{k}^{2} p q \leqq x$ is redundant since $\log m_{k} \leqq e^{k} w(x)$. When $m_{k}, p$, are fixed, $q$ covers a union of at most $3^{\omega\left(m_{k}\right)} \leqq 3^{2 k}$ disjoint intervals with total logarithmic length $\geqq e^{k} /(2 w(x))$. Moreover, all the limit points have logarithm of the order of $e^{k} w(x)$. By the Prime Number Theorem, this implies that the $q$ sum is $\gg w(x)^{-2}$. The $p$-sum is then $\gg 1$ and we have

Now

$$
F_{k} \gg x e^{-k} w(x)^{-3} \sum_{m_{k}} 1 / \varphi\left(m_{k}\right) .
$$

$$
E_{k}^{\prime} \leqq \sum_{m_{k}} \sum_{\substack{a \leq x / m_{k} \\ p \mid a \Rightarrow p\left\lceil m_{k} \text { or } p>r_{k}\right.}} 1 \ll x e^{-k} \sum_{m_{k}} 1 / \varphi\left(m_{k}\right)
$$

again by the sieve. Therefore

$$
F_{k} \gg E_{k}^{\prime} w(x)^{-3}
$$

Now, suppose $E_{M} \gg x$. Then, by (1), $E_{k}^{\prime} \gg E_{k}$ for $L \leqq k \leqq M$, and (2) implies

$$
E_{k+l} \leqq\left(1-c w(x)^{-3}\right) E_{k} \text {. }
$$

Using this inequality recursively, we get

$$
E_{M} \leqq\left(1-c w(x)^{-3}\right)^{[(M-L) / n} E_{L} \ll\left(1-c w(x)^{-3}\right)^{\left[\left(\varepsilon_{1} \log \log x\right) / l\right]} E_{L} .
$$

When $w(x)$ tends to infinity sufficiently slowly, this upper bound is $o(x)$. This is all we need.

\section{Proof of Theorem 2}

Let $\left.\rho>(1-1 / \log 3)^{-1}, J=[(\log \log \log x) / \log \rho)\right]$ and $w(x) \rightarrow \infty, w(x)=o(J)$, be a function to be further specified later. We are going to apply the procedure of the previous section to the numbers

$$
n_{j}=\prod_{\rho^{j}<\log \log p \leqq \rho^{j+1}}^{p \mid n} p,(w(x)<j \leqq J),
$$

for almost all $n \leqq x$. The choice of the $n_{j}$ is heuristically justified by the fact that we certainly need $3^{\omega\left(n_{j}\right)}>\log n_{j}$, and this inequality is normally satisfied if 
$\rho^{j}(\rho-1) \log 3>\rho^{j+1}$ that is $\rho>(1-1 / \log 3)^{-1}$. It will be seen that the following statement holds for any fixed positive $\eta$

$$
\left.\exists d\left|n_{j}, \quad \exists d^{\prime}\right| n_{j}, \quad 0<\left|\log \left(d^{\prime} / d\right)\right|<\eta,(w(x)<j \leqq J), \quad \text { (p.p. } x\right) .
$$

Suppose it is true. Forming all the possible products of $r=[J-w(x)]$ terms by choosing for each $j$ a $d$ or a $d^{\prime}$, we obtain $2^{r}$ distinct divisors of $n$ lying all in an interval of logarithmic length $\eta r$. By Dirichlet's box principle, it follows that

$$
\left.\Delta(n) \geqq 2^{r} / \eta r, \quad \text { (p.p. } x\right) .
$$

This is all that is required.

To establish $(*)$, we define $L_{j}=\left[\rho^{j}(\rho-1-2 \varepsilon)\right], M_{j}=\left[\rho^{j}(\rho-1-\varepsilon)\right]$, and

$$
n_{j, k}=\prod_{\rho^{j}<\log \log p \leqq \rho^{j}+k}^{\left.p\right|_{n}} p, \quad\left(L_{j} \leqq k \leqq M_{j}\right)
$$

where $\varepsilon=\varepsilon(\eta, \rho)$ is positive and sufficiently small.

Using the same arguments as in the proofs of Lemma 4, 5, we easily check that for each $j$ and each $k$ we have

$$
\begin{aligned}
\omega\left(n_{j, k} / n_{j, k-s}\right) & \geqq \alpha s, \quad(\delta \log w(x)<s \leqq k), \quad \text { (p.p. } x) \\
\omega\left(n_{j, k}\right) & \geqq(1-\varepsilon) k, \quad \text { (p.p. } x) \\
\lambda\left(n_{j, k}\right) & \left.\geqq \exp \left(\rho^{j}+k\right) w(x)^{-\delta-3}, \quad \text { (p.p. } x\right)
\end{aligned}
$$

where $1 / \log 3<\alpha<1$, and $\delta=\delta(\alpha)=3 / Q(\alpha)>0$. Moreover, the numbers of exceptional integers are respectively $O\left(x w(x)^{-3}\right), O(x / k)$, and $O\left(x w(x)^{-3}\right)$. The third estimate is achieved by computing an upper bound for

$$
\sum_{n \leqq x} S\left(\theta ; n_{j, k}\right)^{2} z^{\left(\omega\left(n_{j, k}\right)\right.} y^{\omega_{\theta}\left(n_{j}\right)}
$$

with $y=z=1 / 3$ when $\exp \left(-\rho^{j}-k\right) w(x)^{\delta}<|\theta| \leqq \exp \left(-\rho^{j}\right)$, and with $y=1, z=1 / 3$, when $\exp \left(-\rho^{j}\right)<|\theta| \leqq 1 / \eta$. The condition on $\rho$ ensures that the integral on the first range dominates if $\varepsilon$ is small enough. Let $E_{j, k}$ (resp. $E_{j, k}^{\prime}$ ) denote the number of $n \leqq x$ such that

$$
d d^{\prime}\left|n_{j, k}, \quad d \neq d^{\prime} \Rightarrow\right| \log \left(d^{\prime} / d\right) \mid>\eta
$$

(resp. and $\left.\log n_{j, k} \leqq \exp \left(\rho^{j}+\mathrm{k}\right) w(x), \omega\left(n_{j, k}\right) \leqq 2 k, \lambda\left(n_{j, k}\right) \geqq \exp \left(\rho^{j}+k\right) / w(x)^{\delta+3}\right)$. We have

$$
E_{j, k} \leqq E_{j, k}^{\prime}+O\left(x w(x)^{-3}\right)
$$

uniformly for $w(x)<j \leqq J, L_{j} \leqq k \leqq M_{j}$.

Now we may prove exactly like in Sect. 3 that. on the assumption that $E_{j, M} \geqq B \times w(x)^{-3}$ for a sufficiently large absolute constant $B$,

$$
E_{j, k+l} \leqq\left(1-c w(x)^{-\delta-8}\right) E_{j, k}
$$


with $l=1+[\log (4 w(x))]$. Thus

$$
E_{j, M_{3}} \leqq\left(1-c w(x)^{-\delta-8}\right)^{\left[\left(M_{j}-L_{j}\right) / l\right]} x=o\left(x w(x)^{-3}\right),
$$

a contradiction. Finally, it follows that

$$
E_{j, M_{j}} \ll x w(x)^{-3},(w(x)<j \leqq J) .
$$

We have therefore proven that the number of integers $n \leqq x$ which do not satisfy $(*)$ does not exceed

$$
\sum_{w(x)<j \leqq J} E_{j, M_{j}} \ll x J w(x)^{-3}
$$

On choosing $w(x)=\sqrt{\log \log \log x}$, we obtain that this is $o(x)$. This completes the proof.

\section{References}

1. Erdös, P.: On the density of some sequences of integers. Bull. Amer. Math. Soc. 54, 685-692 (1948)

2. Erdös, P., Hall, R.R.: The propinquity of divisors. Bull. London Math. Soc. 11, 304-307 (1979)

3. Erdös, P., Hall, R.R.: On the Möbius function. J. reine angew. Math. 315, 121-126 (1980)

4. Erdös, P., Tenenbaum, G.: Sur la structure de la suite des diviseurs d'un entier. Ann. Inst. Fourier 31, 17-37 (1981)

5. Erdös, P., Tenenbaum, G.: Sur les diviseurs consecutifs d'un entier. Bull. Soc. Math. de France 111, (fasc. 2) $125-145$ (1983)

6. Halberstam, H., Richert, H.-E.: On a result of R.R. Hall. J. Number Theory (1) 11, 76-89 (1979)

7. Hall, R.R.: Sums of imaginary powers of the divisors of the integers. J. London Math. Soc. (2) 9, 571-580 (1975)

8. Hall, R.R., Tenenbaum, G.: Sur la proximité des diviseurs. Recent Progress in Analytic Number Theory, Halberstam, H., Hooley, C. (eds.), vol. 1, 103-113. New York: Academic Press 1981

9. Hall, R.R., Tenenbaum, G.: On the average and normal orders of Hooley's $\Delta$-function. J. London Math. Soc. (2) 25, 392-406 (1982)

10. Hall, R.R., Tenenbaum, G.: The average orders of Hooley's $\Delta_{r}$-functions, Mathematika (to appear)

11. Hooley, C.: On a new technique and its applications to the theory of numbers. Proc. London Math. Soc. (3) 38, 115-151 (1979)

12. Tenenbaum, G.: Lois de répartition des diviseurs 2. Acta Arithm. 38, 1-36 (1980); 3. ibid 39, 19-31 (1981); 4. Ann. Inst. Fourier 29, (3) 1-15 (1979); 5. J. London Math. Soc. (2) 20, 165-176 (1979)

13. Tenenbaum, G.: Sur la proximité des diviseurs. London Math. Soc. Lecture Notes 56, 388-392 (1982)

14. Tenenbaum, G.: Sur la probabilité qu'un entier possède un diviseur dans un intervalle donné Compositio Math. (to appear) 